\title{
Cluster analysis of an international Pressure Pain Threshold database identifies 4 meaningful subgroups of adults with mechanical neck pain
}

David M. Walton PT $\mathrm{PhD}^{1}$, Timothy S. H. Kwok ${ }^{1}$, Swati Mehta PhD(c) ${ }^{1}$, Eldon Loh MD ${ }^{1}$, Ashley Smith PT PhD ${ }^{2}$, James Elliott PT $\mathrm{PhD}^{3}$, Steven J Kamper PT $\mathrm{PhD}^{4}$, Helge Kasch $\mathrm{PhD}^{5}$, Michele Sterling PT $\mathrm{PhD}^{2}$

1: Western University, London Ontario, Canada

2: Griffith University, Southport Queensland, Australia

3: Northwestern University, Chicago Illinois, United States

4: George Institute for Global Health, Sydney New South Wales, Australia

5: Aarhus University, Aarhus, Denmark

Running Head: Pressure Pain Threshold Clusters in Mechanical Neck Pain

The authors declare no conflict of interest.

Correspondence to:

David M. Walton PT, PhD

Assistant Professor, Western University Canada

Rm. EC1443, School of Physical Therapy

1201 Western Rd., London ON

N6G 1H1

Canada

Email: dwalton5@uwo.ca

Phone: 519-661-2111 ext. 80145

Fax: 519-661-3866

Funding Sources: Data for the different databases were collected using funding from the following sources: The Canadian Institutes of Health Research (Canada), The National Institutes of Health (United States), The National Health and Medical Research Council (Australia).

Copyright (C) 2016 Wolters Kluwer Health, Inc. Unauthorized reproduction of the article is prohibited. 
Disclosures: JE is a board member of the Journal of Orthopedic and Sports Physical Therapy and Spine, and holds 35\% ownership in Pain ID LLC. DW is a board member of Manual Therapy and the Journal of Orthopedic and Sports Physical Therapy and is owner of David Walton Rehabilitation Education, Consultation and Research.

Author Contributions:

The following is the description of each author's contribution to the paper:

- David M. Walton: study design, acquisition of data, data analysis and interpretation, drafting of article, final approval

- Timothy S. H. Kwok: data analysis and interpretation, final approval

- Swati Mehta: data analysis and interpretation, final approval

- Eldon Loh: critical revisions, data provision, final approval

- Ashley Smith: acquisition of data, critical revisions, final approval

- James Elliott: acquisition of data, critical revisions, final approval

- Steven J Kamper: acquisition of data, critical revisions, final approval

- Helge Kasch: acquisition of data, critical revisions, final approval

- Michele Sterling: acquisition of data, critical revisions, final approval

\section{INTRODUCTION}

Measurement for Pressure Pain Detection Threshold (PPDT) is increasing in value as clinical practice incorporates Quantitative Sensory Testing (QST) into pain evaluation. There is increasing evidence that QST provides valuable information regarding the nature of a pain experience and potential mechanisms beyond that provided by self-reports of pain intensity. ${ }^{1-3}$ Kasch and colleagues ${ }^{4}$ provided evidence that PPDT offers some prognostic value as a risk factor for chronicity in acute whiplash-associated disorder (WAD), which was independently verified by Walton and colleagues. ${ }^{5}$

A common approach to evaluating PPDT in neck pain is to capture values at a local site (about the neck) and an anatomically distal site such as the tibialis anterior (TA) muscle belly in the lower leg. While the distal site may have been originally intended as a form of 'control', emerging evidences suggests it is an important test on its own. For example, globally low PPDT, indicating high sensitivity to blunt pressure, may be a risk factor for the subsequent transition to persistent problems ${ }^{4,5}$ and may be a clinical proxy for serological markers in musculoskeletal conditions such as osteoarthritis. ${ }^{3}$ Widespread low PPDT has been observed in several conditions including $\mathrm{WAD}^{6}$, lateral epicondylalgia ${ }^{7}$, carpal tunnel syndrome ${ }^{8}$, and chronic headache. ${ }^{9}$ The precise mechanisms underlying global mechanical hyperalgesia are yet unclear, but may indicate sensitization of central nociceptive processing pathways that are unlikely to quickly respond to conservative therapies. ${ }^{1}$

Anecdotal evidence indicates that patients with neck pain are likely to fall into one of 3 categories: average sensitivity, locally hypersensitive (sensitive at the neck but not the TA), or globally hypersensitive (sensitive at both the neck and TA), each of which may lead to a different treatment or prognostic decision. There is evidence that global hyposensitivity may also exist and represent a $4^{\text {th }}$ subgroup $^{10,11}$, but this is more commonly associated with electrical, thermal and vibratory perception thresholds than it is with pain threshold, and the available evidence suggests it is unique to WAD. ${ }^{11}$ 
To date the majority of empirical evidence supporting PPDT provides results as correlations or group mean differences. While useful for identifying associations, correlational data is less useful for clinical decisions regarding individual patients. There are few examples of evidence-guided phenotyping using PPDT as the predictor variable. ${ }^{12}$ If PPDT is to enter routine clinical use for prognostic, treatment, or outcomes decisions in neck pain then clinicians need a better understanding of how it can be used to classify patients and the clusters that exist in this population. The purpose of this study was to conduct a pooled secondary analysis of large international databases of people with mechanical neck pain (MNP) using latent class (cluster) analysis to identify clinically meaningful subgroups within the sample as a first step towards translating existing PPDT knowledge into more accessible clinical practice.

\section{METHODS}

This was a secondary analysis of 5 databases of people with mechanical neck pain who were either recruited specifically for research purposes, or who presented for medical or rehabilitation intervention for their neck pain problem. The databases included participants from Canada $(n=403)$, the United States $(n=79)$, Denmark $(n=432)$, and Australia $(n=262)$. Data were collected either between 20012004 or between 2007 and 2014. Institutional Review Board approval was obtained for each cohort individually prior to data collection. A total of 1176 adults formed the collective database. While inclusion/exclusion criteria differed slightly between the databases, the common inclusion criteria were: age 18 years or older, free of serious systemic disease (including cancer, neuromuscular disease, advanced organ failure), have no evidence of cognitive deficit, and able to read and understand the local language at a minimum grade 6 level. The neck pain was not due to tumour, fracture or dislocation, but could have been the result of trauma, posture, degenerative or other insidious (unknown) causes as long as it was deemed mechanical in nature. Participants were not excluded due to the presence of radicular signs (radiating pain, numbness), medicolegal or compensation status. Some databases focused exclusively on acute neck pain, some on chronic, and others mixed acute and chronic. In all cases participants were not excluded if pain was present in other body regions (save for pain in the anterior lower leg) but neck pain had to be the predominant complaint.

Data collection procedures were variable in timing and order but similar PPDT collection allowed pooled analysis. Subjects across all databases completed an intake questionnaire that provided information on basic demographics (age, sex) and the nature of the condition (cause and duration of neck pain). Pressure Pain Detection Thresholds were recorded from all subjects at both a local site (e.g. about the C5/6 lateral articulation) and a distal site (TA muscle belly). PPDT were collected in a similar format across all databases, either bilaterally or over the most sensitive side only. In all cases, the participants were seated or prone for neck evaluation or lying in a crook supine position for TA with the area to be tested exposed. A digital algometer with a rubber probe of $1 \mathrm{~cm}^{2}$ was applied over the region to be tested a steadily increasing rate of $4-5 \mathrm{~N}$ (40-50 kPa or 0.4-0.5 kgf)/s. Subjects were instructed to either press a button (Somedic Algometer, Somedic AB Sweden) or tell the examiner the moment the sensation changed from one of pressure to one of pain (Wagner FDX 25, Wagner Instruments USA). A minimum of 30 seconds was observed between tests, and in each case the test was repeated 3 times in each region. Subsequent components of each research study differed, but only the standardized PPDT, self-report scales, and demographic data were extracted for this analysis. 
Not all cohorts included the same clinical measures. The Neck Disability Index ${ }^{13}$ (NDI) or Copenhagen Neck Functional Disability Index ${ }^{14}$ (combined $\left.n=973,0-100 \%\right)$ and 0-10 Numeric Pain Rating Scale ${ }^{15}$ (NPRS, $n=1170$ ) were completed by the majority of subjects in all databases. Other tools that were completed by substantial numbers of participants included: the Tampa Scale of Kinesiophobia ${ }^{16}$ (TSK, range 0-44, $\mathrm{n}=336$ ), the Pain Catastrophizing Scale ${ }^{17}$ (PCS, range 0-52, $\mathrm{n}=389$ ), the Impact of Events Scale $^{18}$ (IES, range 0-45, $\mathrm{n}=511$ ), and the Self-report version of the Leeds Assessment of Neuropathic Signs and Symptoms ${ }^{19}$ (SLANSS, range 0-24, $\mathrm{n}=408$ ). Respectively, these tests are intended to capture fear of movement/(re)injury, exaggerated negative orientation towards pain, post-traumatic stress symptoms, and neuropathic signs and symptoms. All have documented evidence of adequate validity for use in neck pain. ${ }^{20-23}$ Active range of motion was captured in 174 participants using a digital inclinometer and reported as total mobility in the sagittal plane (absolute cervical flexion + extension) and total mobility in the frontal plane (absolute left sidebend + right sidebend).

\section{ANALYSIS}

The means of the repeated tests at each of the most sensitive neck and TA sites were calculated for each subject. Recognizing that this was a secondary analysis of databases from different research groups in different locations, some non-random (systematic) bias was expected due to variation in instructions, contexts and/or testing procedures. It is also well-recognized that males and females show systematic differences in PPDT, with females on average scoring lower than males. ${ }^{5,24}$ To address this, each cohort was first stratified by gender before PPDT was converted to a Z-score for each subject by the formula: (Individual PPDT- (cohort gender-specific mean PPDT))/cohort gender-specific SD using Microsoft Excel 2013 (Microsoft Corp., Seattle). In this way, each participant's score was converted to a unitless number indicating the number of standard deviations away from the mean for that gender in that cohort, with negative values being those that fell below the mean while positive values were above the mean. In those cohorts that recorded PPDT bilaterally only the values from the most sensitive side were used. Those subjects falling 5 or more standard deviations from the mean were considered outliers for the purpose of this analysis and were removed.

Descriptive statistics (means $+/-\mathrm{SD}$, frequencies, ratios) were calculated for the demographic and subject characteristics. Simple bivariate correlations using Pearson's $r$ were explored between PPDT and each of the secondary (predictor) outcomes to allow comparisons with previous literature. A Latent Profile Analysis was undertaken using the $\mathrm{K}$ means cluster approach as described by DiStefano and Kamphaus. ${ }^{25}$ This approach is intended to identify non-overlapping clusters (denoted as ' $k$ ') based on the Euclidean distance between individual data points by testing serially increasing numbers of clusters against predefined fit criteria in comparison to k-1 clusters. ${ }^{26}$ The independent (predictor) variables were ztransformed PPDT at the local (neck) site and distal (TA) site. A satisfactory model solution could not be found with the entire mixed-gender cohort, so models were constructed for males and females independently. The k-means approach starts with a base 2-cluster model (high or low across all predictors) and calculates a series of fit indicators to describe how well the data fit the 2-cluster solution. The fit indicators of interest were the Bayesian Information Criterion (BIC), Akaike Information Criterion (AIC), Entropy, and the Lo-Mendell-Rubin Likelihood Ratio Test (LMR-LRT). While no set criteria exist for 'good' fit, the general approach is to accept the model with the lowest BIC and AIC, and highest 
entropy (closest to 1.0). The LMR-LRT provides a statistical comparison of the fit of the k vs. k-1 cluster solutions. A significant $(<0.05) \mathrm{p}$ value indicates that the $\mathrm{k}$ cluster solution is favoured over the $\mathrm{k}-1$ solution. Additional solutions are tested until the fit indices no longer indicate statistical or theoretical advantage of the k cluster solution, in which case the k-1 solution (being the last solution that provided significant improvement over previous) is generally accepted for reasons of parsimony. Analysis was conducted using MPlus software v6.12.

The empirically-derived clusters were then explored for meaningful differences in independent variables using SPSS v23 (IBM Corp.). Locations of each cluster on each variable, relative to the mean of 0.00 , was explored using the z-test to determine statistical difference from the mean. One-way independent Analysis of Co-Variance (ANCOVA) with gender as the covariate, was used to compare inter-cluster differences in Z-scores with a Bonferroni-corrected post-hoc test $(\mathrm{p}=0.0125)$ when significant main or interaction effects were found. For this analysis, AROM was combined to create a global AROM score (flexion + extension + left + right sidebend $)$.

\section{RESULTS}

Table 1 presents the characteristics of the combined database. It was $70.9 \%$ female with a mean duration of symptoms of 23.8 months and a modal etiology of whiplash-associated disorder (WAD). Age ranged from 18 to 71 years. Mean (SD) pain intensity of the overall sample was 4.9/10 (2.2) with a range from 0 to 10 . Mean (SD) NDI score was $37.4 \%(17.5 \%)$ with a range from 0 to 100 . Table 2 presents the results of simple bivariate correlations between PPDT at the two locations and the descriptor variables included in the cohorts. Local PPDT showed small-to-moderate significant positive correlations with cervical AROM, and negative correlations with all variables except IES score. Distal PPDT showed small $(<0.22)$ but significant negative correlations with NDI, TSK, NRS, and IES scores.

Table 3 presents the fit indicators for the different cluster models tested in both males and females. In males, support was found for the 3, 4 or 5 cluster solutions. In females, the 4 cluster solution was most supported. For purposes of optimizing clinical translation, a 4-cluster solution was accepted for both sexes (Table 4, Figure 1). The 4 male clusters, described by relative $\mathrm{Z}$ scores at the local-distal sites, were: Low-Low (46.5\%), Moderate-Moderate (Mod-Mod, 38.5\%), Mod-High (5.3\%) and High-High (9.7\%). In the females the clusters were: Low-Low (74.9\%), Mod-Mod (20.1\%), Mod-High (3.6\%) and High-High PPDT (1.4\%). For translation purposes, Table 4 also includes absolute PPDT values (kPa) for one of the cohorts in the main database $(n=310)$. ANCOVA supported the non-overlapping nature of these clusters, with significant differences existing between all 4 at both local and distal sites via Bonferroni post-hoc tests $(\mathrm{p}<0.0125)$.

Table 5 presents intracluster analyses via Z-test. These analyses revealed that the Low-Low cluster was the only of the 4 clusters to have a mean Z-score significantly above the mean (worse) for each of NDI, NRS, and PCS scores and significantly below the mean (worse) in AROM. The Mod-Mod group was significantly below the mean (better) on the same 3 self-report tools, and significantly above the mean (better) in AROM. The High-High cluster was also significantly below the mean (better) for NDI, and was the only cluster significantly below the mean (better) for TSK scores. Inter-cluster ANCOVA-based comparisons revealed that after controlling for the effect of sex, significant main effects of cluster existed 
for NDI ( $\mathrm{F}=18.00, \mathrm{p}<0.01)$, NRS $(\mathrm{F}=9.26, \mathrm{p}<0.01)$, and PCS $(\mathrm{F}=5.37, \mathrm{p}<0.01)$ scores. Bonferronicorrected post-hoc tests revealed significantly lower scores on the NDI, NRS and PCS in the Mod-Mod cluster compared to the Low-Low cluster (all p<0.01). AROM was significantly higher in the Mod-Mod cluster compared to the Low-Low cluster $(\mathrm{p}<0.01)$. No other inter-cluster differences reached statistical significance.

\section{DISCUSSION}

The results of a latent profile analysis revealed 4 meaningful clusters that are statistically and clinically justifiable. The results show potentially meaningful clinical differences between the clusters, especially the two largest clusters (Low-Low and Mod-Mod). This study also identified two smaller clusters which have not been described in previous literature on the subject in neck pain (Mod-High, High-High).

The characteristics of the subjects in this study, primarily female with mean age of near 40, and NPRS of 4.9/10 are generally comparable with previous large samples in MNP studies. ${ }^{27,28}$ The mean duration of approximately 2 years and NDI score of 37/100 suggests these results are most generalizable to those with chronic MNP of moderate pain-related disability. ${ }^{13}$

The most comparable published data to these results are those of Pedler and Sterling ${ }^{29}$ who identified 4 clusters of people with chronic WAD using sensory hypersensitivity and post-traumatic stress symptoms as the clustering variables in an independent sample. Those authors considered PPDT scores as a combined 'sensitivity' variable, while we interpreted the two regions separately. Similar to our results, Pedler and Sterling ${ }^{29}$ found statistical support for both the 3- and 4-cluster solutions and endorsed the 4 cluster solution by virtue of clinical utility. It is unknown whether our cluster solution would have been improved through consideration of an additional psychological variable. The German Research Network on Neuropathic Pain ${ }^{30}$ have reported on clusters of QST responses in 1236 patients with different neuropathic pain syndromes, similarly using Z-transformed scores to address systematic differences across cohorts. That group included standardized multimodal QST measures where we focused only on blunt mechanical pressure thresholds. There are few intuitive comparisons that can be drawn between these two databases given the type and format of data presented and differences in pain aetiology.

The clusters may be of value from mechanistic or prognostic perspectives. Global hypersensitivity in PPDT (Low-Low) is thought to be a proxy for disordered central nociceptive processing. ${ }^{31}$ As an example, Ge and colleagues ${ }^{32}$ employed PPDTs of the neck and the tibialis anterior as clinical indications of descending noxious inhibitory control following fatiguing muscle contractions. They reported differing responses in PPDT between healthy controls and subjects with fibromyalgia syndrome (FMS) following the contraction. These authors therefore postulated that the decrease in PPDT at the distal (TA) site was an indication of disordered descending control. Lemming and colleagues ${ }^{33}$ similarly postulated disordered central nociceptive processing through use of a saline x pressure cuff protocol. Their findings, showing a significantly greater increase in pressure sensitivity in a group with chronic WAD compared to healthy controls, supported their position of a central processing problem in this population. Ashina and colleagues $^{34}$ used PPDT at the UFT and TA sites to provide evidence of disordered nociceptive processing in 16 patients with chronic tension-type headache suggesting possible application of these findings outside of MNP. Global mechanical hypersensitivity in acute WAD may also be useful as a risk 
factor for the subsequent transition to chronic $\mathrm{WAD}^{5}$ although this effect may be removed when the effects of other variables are controlled. ${ }^{35}$ Our intracluster secondary variable analyses provide some clues as to potential mechanisms if PPDT does function as a prognostic indicator. The Low-Low cluster was the only one with NDI, PCS and NRS scores higher than the cohort-specific mean values. NDI, PCS, and NRS have each demonstrated consistent prognostic value in acute WAD. ${ }^{23}$ It is therefore possible that PPDT is serving as a proxy for these other variables, or vice versa, or that more likely the relationship is more complex representing mediating or moderating relationships. Multivariate analyses using longitudinal models would be required to address the independent utility of each.

It is tempting to suggest that the Mod-High cluster, demonstrating relatively greater sensitivity at the local than distal sites, represents those with a local inflammatory process or other impairment but otherwise spared nociceptive processing elsewhere. While this is only speculation, it may indicate the potential to use PPDT clustering with self-report scores to triangulate clinical findings for a more robust estimate of a patient's risk or mechanistic profile.

The High-High group is small, with both local and distal mean PPDT values at least 1.5 SD above the mean. Baumgartner and colleagues ${ }^{36}$ have provided evidence of a subgroup of patients with neuropathic pain who present with 'painful hypoalgesia', which may be occurring here as well. However, we are cautious in inferring mechanistic interpretation to this cluster, as the lower TSK score was the only secondary predictor variable to show any statistical deviation from the mean. Whether this represents a small cluster of individuals presenting with mechanical neck pain who are otherwise stoic in the face of painful stimuli, or this represents a small cluster in whom the nociceptive system is in a state of relative hyposensitivity is impossible to discern at this time.

Bivariate correlations were all in the expected direction but were of small to moderate magnitude. Kamper and colleagues ${ }^{37}$ reported similar magnitude of association between NPRS and PPDT $(r=-0.20$ to -0.33 , $\mathrm{cf}-0.18$ in the current study). Rivest and colleagues ${ }^{38}$ reported a correlation of $\mathrm{r}=-0.42$ between local PPDT and NDI score in a sample of 37 subjects with subacute WAD, comparable to the $r=-0.30$ in our sample of mixed neck pain. Wallin and colleagues ${ }^{39}$ reported a small association between PPDT and PCS scores in 28 subjects with chronic WAD, however their relationship failed to reach statistical significance in multivariate regression. Similar findings were reported by Rivest and colleagues ${ }^{38}$ in their sample ( $r=-0.22$ with PCS, n.s. $\mathrm{cf} r=-0.19$ herein). Interpretation of the secondary variable analyses is that PPDT may have a small association with some measures of cognitive distress (i.e. catastrophizing, fear of movement, emotional distress) but there are likely other mechanisms that play a larger role.

One notable result worthy of discussion is the Impact of Events Scale, intended to quantify the magnitude of event related emotional distress. ${ }^{18}$ While the correlation with local and distal PPDT were both in the same direction, only the magnitude of the distal correlation reached statistical significance. Intercluster comparisons were not statistically significant for IES, meaning inferences should be made cautiously. Association between the distal site and IES is potentially intriguing, and may suggest a shared mechanism or may be statistical artifact. Whether this indicates a small but potentially meaningful phenotype of people with heightened post-traumatic stress symptoms and generalized mechanical hyperalgesia, such as that shown by Pedler and Sterling ${ }^{30}$ remains to be seen but represents an interesting area for further study. 
Some limitations to interpretation include the small absolute $\mathrm{N}$ in each of the smaller two clusters and the inability to assign any true mechanistic properties to the clusters. The secondary nature of the data analysis, including a combination of 5 separate databases with systematic differences in sample characteristics and data collection may improve generalizability while threatening internal validity. One of the earlier steps in this analysis was to compare mean PPDT values across all 5 databases, and as expected there were significant differences; 1 cohort comprising entirely subjects with chronic MNP showed higher mean local and distal PPDT, and 1 cohort of mixed MNP showed lower mean local PPDT (comparison not shown). As a result, the Z-transformation procedure was undertaken to abolish systematic differences between cohorts by assigning each subject a unitless relative value. While we believe this is a sound method of addressing the threat to internal validity, it also introduces an additional challenge to translation of these findings in that $\mathrm{z}$ scores are difficult to interpret in isolation. Fortunately, some normative databases have been published ${ }^{5}$ that clinicians can access to determine where their patient lies relative to the expected mean for that population, and therefore estimate to which of the 4 clusters the patient likely belongs. Sample threshold values for each cluster in $\mathrm{kPa}$ drawn from one of the larger clusters have also been provided in Table 4. Naming of the clusters was subjective and based on the patterns of PPDT responses within each, but no a priori thresholds for Low/Moderate/High were set. One final limitation is that the entropy statistic, as an indicator of classification accuracy, was lower than desired in all of the cluster solutions. Entropy is one of 4 fit indices employed herein, an estimator of classification accuracy that appears to be more relevant for categorical rather than interval-level data. ${ }^{40}$ The simple interpretation is that there may yet exist additional subgroups or stratification variables beyond gender that are affecting classification accuracy and thereby adversely influencing the entropy statistic. The decision to accept a 4-cluster solution in both genders was informed by statistical as well as clinical considerations, however a 3 - or 5-cluster solution was also defendable for the male subjects.

Identifying the clusters that appear to exist in this population is just one step towards improved management. Next steps would be to independently verify these clusters in a separate sample, then to explore the potential value of these clusters for intervention decisions. Exploratory (mechanistic) studies may also be required as an intervening step between independent verification and intervention trials in order to identify the most appropriate treatment target. The current study facilitates this research direction, and while independent verification is urged, we are confident that the clusters presented here are both statistically and clinically meaningful. 


\section{Reference List}

1. Giesecke, J., Reed, B.D., Haefner, H.K., Giesecke, T., Clauw, D.J., Gracely, R.H. Quantitative sensory testing in vulvodynia patients and increased peripheral pressure pain sensitivity. Obstet. Gynecol. 2004;104,126-133.

2. Siegenthaler, A., Eichenberger, U., Schmidlin, K., Arendt-Nielsen, L., Curatolo, M. What does local tenderness say about the origin of pain? An investigation of cervical zygapophysial joint pain. Anesth. Analg. 2010;110, 923-927.

3. Arendt-Nielsen, L., Egsgaard, L.L., Petersen, K.K., Eskehave, T.N., Graven-Nielsen, T., Hoeck, H.C., Simonsen, O. A mechanism-based pain sensitivity index to characterize knee osteoarthritis patients with different disease stages and pain levels. Eur. J. Pain 2014;19, 1406-1417.

4. Kasch, H., Qerama, E., Kongsted, A., Bach, F.W., Bendix, T., Jensen, T.S. Deep muscle pain, tender points and recovery in acute whiplash patients: a 1-year follow-up study. Pain 2008;140, 65-73.

5. Walton, D., MacDermid, J., Nielson, W., Teasell, R., Reese, H., Levesque, L. Pressure pain threshold testing demonstrates predictive ability in people with acute whiplash. JOSPT 2011;41, 658-665.

6. Scott, D., Jull, G., Sterling, M. Widespread sensory hypersensitivity is a feature of chronic whiplashassociated disorder but not chronic idiopathic neck pain. Clin. J. Pain 2005;21, 175-181.

7. Fernandez-Carnero, J., Fernandez-de-Las-Penas, C., de la Llave-Rincon, A.I., Ge, H.Y., ArendtNielsen, L. Widespread mechanical pain hypersensitivity as sign of central sensitization in unilateral epicondylalgia: a blinded, controlled study. Clin. J. Pain 2009;25, 555-561.

8. Fernandez-de-las-Penas, C., de la Llave-Rincon, A.I., Fernandez-Carnero, J., Cuadrado, M.L., Arendt-Nielsen, L., Pareja, J.A. Bilateral widespread mechanical pain sensitivity in carpal tunnel syndrome: evidence of central processing in unilateral neuropathy. Brain 2009;132, 1472-1479.

9. Uthaikhup, S., Sterling, M., Jull, G. Widespread sensory hypersensitivity is not a feature of chronic headache in elders. Clin. J. Pain 2009;25, 699-704.

10. Chien, A., Eliav, E., Sterling, M. Hypoesthesia occurs in acute whiplash irrespective of pain and disability levels and the presence of sensory hypersensitivity. Clin. J. Pain 2008;24, 759-766.

11. Chien, A., Eliav, E., Sterling, M. Hypoaesthesia occurs with sensory hypersensitivity in chronic whiplash - Further evidence of a neuropathic condition. Man. Ther. 2009;14, 138-146.

12. Kasch, H., Qerama, E., Kongsted, A., Bach, F.W., Bendix, T., Jensen, T.S. The risk assessment score in acute whiplash injury predicts outcome and reflects biopsychosocial factors. Spine 2011;36, S2637.

13. Vernon, H., Mior, S. The Neck Disability Index: a study of reliability and validity. J. Manipulative Physiol. Ther. 1991;14, 409-415.

14. Jordan, A., Manniche, C., Mosdal, C., Hindsberger, C. The Copenhagen Neck Functional Disability Scale: a study of reliability and validity. J. Manipulative Physiol. Ther. 1998;21, 520-527.

15. Jensen, M.P., Karoly, P., Braver, S. The measurement of clinical pain intensity: a comparison of six methods. Pain 1986;27, 117-126.

16. Vlaeyen, J.W., Kole-Snijders, A.M., Boeren, R.G., van Eek, H. Fear of movement/(re)injury in chronic low back pain and its relation to behavioral performance. Pain 1995; 62, 363-372.

17. Sullivan, M.J.L., Bishop, S.R., Pivik, J. The Pain Catastrophizing Scale: Development and Validation. Psychological Assessment 1995; 7, 524-532.

18. Horowitz, M., Wilner, N., Alvarez, W. Impact of Event Scale: a measure of subjective stress. Psychosom. Med.1979; 41, 209-218. 
19. Bennett, M.I., Smith, B.H., Torrance, N., Potter, J. The S-LANSS score for identifying pain of predominantly neuropathic origin: validation for use in clinical and postal research. J. Pain 2005;6, 149-158.

20. Sterling, M., Kenardy, J., Jull, G., Vicenzino, B. The development of psychological changes following whiplash injury. Pain 2003; 106, 481-489.

21. Sterling, M., Pedler, A. A neuropathic pain component is common in acute whiplash and associated with a more complex clinical presentation. Man. Ther. 2009;14, 173-179.

22. Walton, D., Elliott, J. A Higher-Order Analysis Supports Use of the Tampa Scale of Kinesiophobia 11-Item Version in People With Neck Pain. Phys. Ther. 2012;93, 60-68.

23. Walton, D.M., Wideman, T.H., Sullivan, M.J. A Rasch analysis of the pain catastrophizing scale supports its use as an interval-level measure. Clin. J. Pain 2013;29, 499-506.

24. Binderup, A.T., Arendt-Nielsen, L., Madeleine, P. Pressure pain sensitivity maps of the neck-shoulder and the low back regions in men and women. BMC Musculoskelet. Disord. 2010; 11, 234.

25. DiStefano, C., Kamphaus, R.W. Investigating subtypes of child development: a comparison of cluster analysis and latent class cluster analysis in typology creation. Educ. Psychol. Meas. 2006;66, 778794.

26. Tan, P.N., Steinbach, M., Kumar, V. Introduction to data mining. USA, Boston: Addison-Wesley. 2005.

27. Michaleff, Z.A., Maher, C.G., Lin, C.W., Rebbeck, T., Jull, G., Latimer, J., Connelly, L., Sterling, M. Comprehensive physiotherapy exercise programme or advice for chronic whiplash (PROMISE): a pragmatic randomised controlled trial. Lancet 2014;384, 133-141.

28. Seferiadis, A., Ohlin, P., Billhult, A., Gunnarsson, R. Basic body awareness therapy or exercise therapy for the treatment of chronic whiplash associated disorders: a randomized comparative clinical trial. Disabil. Rehabil. 2015;1-10.

29. Pedler, A., Sterling, M. Patients with chronic whiplash can be subgrouped on the basis of symptoms of sensory hypersensitivity and posttraumatic stress. Pain 2013;154, 1640-1648.

30. Maier, C., Baron, R., Tolle, T.R., Binder, A., Birbaumer, N., Birklein, F., Gierthmuhlen, J., Flor, H., Geber, C., Huge, V., et al. Quantitative sensory testing in the German Research Network on Neuropathic Pain (DFNS): somatosensory abnormalities in 1236 patients with different neuropathic pain syndromes. Pain 2010;150, 439-450.

31. Petersel, D.L., Dror, V., Cheung, R. Central amplification and fibromyalgia: disorder of pain processing. J. Neurosci. Res. 2011;89, 29-34.

32. Ge, H.Y., Nie, H., Graven-Nielsen, T., Danneskiold-Samsoe, B., Arendt-Nielsen, L. Descending pain modulation and its interaction with peripheral sensitization following sustained isometric muscle contraction in fibromyalgia. Eur. J. Pain 2012;16, 196-203.

33. Lemming, D., Graven-Nielsen, T., Sorensen, J., Arendt-Nielsen, L., Gerdle, B. Widespread pain hypersensitivity and facilitated temporal summation of deep tissue pain in whiplash associated disorder: an explorative study of women. J. Rehabil. Med. 2012;44, 648-657.

34. Ashina S, Babenko L, Jensen R, Ashina M, Magerl W, Bendtsen L. Increased muscular and cutaneous pain sensitivity in cephalic region in patients with chronic tension-type headache. Eur J Neurol. 2005 Jul;12(7):543-9.

35. Sterling, M., Hendrikz, J., Kenardy, J. Similar factors predict disability and posttraumatic stress disorder trajectories after whiplash injury. Pain 2011;152, 1272-1278 
36. Baumgartner, U., Magerl, W., Klein, T., Hopf, H.C., Treede, R.D. Neurogenic hyperalgesia versus painful hypoalgesia: two distinct mechanisms of neuropathic pain. Pain 2002;96, 141-151.

37. Kamper, S.J., Maher, C.G., Hush, J.M., Pedler, A., Sterling, M. Relationship between pressure pain thresholds and pain ratings in patients with whiplash-associated disorders. Clin. J. Pain 2011;27, 495501.

38. Rivest, K., Cote, J.N., Dumas, J.P., Sterling, M., De Serres, S.J. Relationships between pain thresholds, catastrophizing and gender in acute whiplash injury. Man. Ther. 2010;15, 154-159.

39. Wallin, M., Liedberg, G., Borsbo, B., Gerdle, B. Thermal detection and pain thresholds but not pressure pain thresholds are correlated with psychological factors in women with chronic whiplashassociated pain. Clin. J. Pain 2012;28, 211-221.

40. Celeux, G., Soromenho, G. An entropy criterion for assessing the number of clusters in a mixture model. J Classification 1996;13, 195-212.

Figure 1. Relative locations of the 4 clusters identified in males (above) and females (below). Solid line = Low-Low; Dotted line $=$ Moderate-Moderate; Dashed line $=$ High-High; Dotted-Dashed line $=$ Moderate-High.

Table 1. Descriptions of the sample, combined and separated by country.

\begin{tabular}{|lllllll|}
\hline & Overall & \multicolumn{1}{c}{$\mathbf{1}$} & \multicolumn{1}{c}{$\mathbf{2}$} & \multicolumn{1}{c}{$\mathbf{3}$} & \multicolumn{1}{c|}{$\mathbf{4}$} & \multicolumn{1}{c|}{$\mathbf{5}$} \\
\hline Country & N/A & Canada & Canada & USA & Australia & Denmark \\
\hline $\mathbf{N}$ & 1176 & 85 & 318 & 79 & 262 & 432 \\
\hline \% Female & 71 & 67 & 74 & 100 & 63 & 69 \\
\hline $\begin{array}{l}\text { \% Litigating/ } \\
\text { Receiving } \\
\text { Compensation }\end{array}$ & 38 & 53 & 8 & 65 & $\begin{array}{l}\text { Not } \\
\text { available }\end{array}$ & $\begin{array}{l}\text { Not } \\
\text { available }\end{array}$ \\
\hline Age (mean yrs, SD) & $38(13)$ & $45(10)$ & $42(13)$ & $30(8)$ & $40(13)$ & $35(12)$ \\
\hline $\begin{array}{l}\text { Duration in months } \\
\text { (Mean, range) }\end{array}$ & $24(0$ to & $\begin{array}{l}59(9 \text { to } \\
\text { 580) }\end{array}$ & $\begin{array}{l}31(0 \text { to } \\
\text { 580) }\end{array}$ & $\begin{array}{l}\text { 20 (3 } \\
\text { to 36) }\end{array}$ & $8(0$ to 67) & $<1 \mathrm{mth}$ \\
\hline
\end{tabular}


Table 2. Bivariate correlations (Pearson's $r$ ) between PPDT and predictor variables. *: correlation is significant at the $\mathrm{p}<0.05$ level. **: correlation is significant at the $\mathrm{p}<0.01$ level.

PPDT $=$ Pressure pain detection threshold, AROM = Active range of motion, NDI $=$ Neck Disability Index, CNFDI = Copenhagen Neck Functional Disability Index, TSK = Tampa Scale for Kinesiophobia, PCS = Pain Catastrophizing Scale, NPRS = Numeric Pain Rating Scale, SLANSS = Self-report version of Leeds Assessment of Neuropathic Signs and Symptoms, IES = Impact of Events Scale.

\begin{tabular}{|l|l|l|}
\hline Variable (n) & Local PPDT & \multicolumn{1}{l|}{ Distal PPDT } \\
\hline Local PPDT min (1176) & & $0.60^{* *}$ \\
\hline NDI/CNFDI (973) & $-0.30^{* *}$ & $-0.21^{* *}$ \\
\hline TSK (337) & $-0.16^{* *}$ & $-0.11^{*}$ \\
\hline PCS (378) & $-0.19^{* *}$ & -0.08 \\
\hline NPRS (1170) & $-0.18^{* *}$ & $-0.09^{*}$ \\
\hline SLANSS (420) & $-0.15^{* *}$ & -0.09 \\
\hline IES total (79) & -0.15 & $-0.20 *$ \\
\hline AROM sagittal (174) & $0.29 * *$ & 0.09 \\
\hline AROM frontal (174) & $0.31^{* *}$ & 0.12 \\
\hline
\end{tabular}

Table 3. Fit indicators for the different cluster solutions tested. Fit indicators for the tested cluster solutions for males $(n=340)$ and females $(n=833)$. AIC $=$ Aikake Information Criterion, BIC $=$ Bayesian Information Criterion, LMR-LRT = Lo-Mendel-Rubin Likelihood Ratio Test. In both cases, the 5 and 6 cluster solutions showed neither statistical nor clinical meaningfulness.

\begin{tabular}{|l|l|l|l|l|l|}
\hline & AIC & BIC & Entropy & $\begin{array}{l}\text { LMR-LRT } \\
(\mathbf{p})\end{array}$ & $\begin{array}{l}\text { Classification } \\
\text { accuracy }\end{array}$ \\
\hline \multicolumn{6}{|l|}{} \\
\hline 2 Classes & 2037.2 & 2064.3 & 0.73 & $128.0(0.00)$ & $86.4-93.6$ \\
\hline 3 Classes & 2008.3 & 2047.0 & 0.74 & $33.0(0.01)$ & $83.5-91.2$ \\
\hline 4 Classes & 1986.3 & 2036.6 & 0.76 & $26.5(0.09)$ & $80.2-91.7$ \\
\hline 5 Classes & 1894.2 & 1955.4 & 0.80 & $17.9(0.04)$ & $79.1-91.3$ \\
\hline 6 Classes & 1895.6 & 1968.4 & 0.74 & $4.3(0.78)$ & $66.5-89.9$ \\
\hline \multicolumn{7}{|l|}{} & $322.4(0.00)$ & $89.6-96.4$ \\
\hline 2 Classes & 3905.6 & 3938.7 & 0.83 & $91.0(0.04)$ & $83.9-95.1$ \\
\hline 3 Classes & 3816.1 & 3863.3 & 0.84 & $99.9(0.00)$ & $82.0-94.7$ \\
\hline 4 Classes & 3717.2 & 3778.6 & 0.75 & $26.2(0.24)$ & $78.4-95.5$ \\
\hline 5 Classes & 3695.6 & 3771.2 & 0.79 & $19.2(0.89)$ & $76.5-91.7$ \\
\hline 6 Classes & 3681.3 & 3771.0 & 0.78 &
\end{tabular}

Copyright (C) 2016 Wolters Kluwer Health, Inc. Unauthorized reproduction of the article is prohibited. 
Table 4. Cluster characteristics and relative proportions of the overall sample. The values are mean $\mathrm{Z}$ score $(95 \%$ confidence interval) for each cluster. Overall sample mean $=0.00, \mathrm{SD}=1.00$. PPDT $=$ pressure pain detection threshold. Differences in $\mathrm{Z}$ scores between the clusters are all significant at the $\mathrm{p}<0.05$ level by virtue of non-overlapping confidence intervals. $\mathrm{kPa}=$ absolute value in kilopascals for one cohort $(\mathrm{n}=310)$.

\begin{tabular}{|lllll|}
\hline Cluster & & Local PPDT & Distal PPDT & $\begin{array}{l}\text { Percent of } \\
\text { sample }\end{array}$ \\
\hline $\mathbf{1}$ & Level & Low & Low & $67 \%$ \\
& $\mathrm{Z}$ score $(95 \% \mathrm{CI})$ & $-0.52(-0.56$ to -0.48$)$ & $-0.48(-0.53$ to -0.44$)$ & \\
& $\mathrm{kPa}(95 \% \mathrm{CI})$ & $244.7(221.4$ to 268.0$)$ & $367.1(333.4$ to 400.8$)$ & \\
\hline $\mathbf{2}$ & Level & Moderate & Moderate & $25 \%$ \\
& $\mathrm{Z} \mathrm{score}(95 \% \mathrm{CI})$ & $0.89(0.83$ to 0.95$)$ & $0.48(0.41$ to 0.54$)$ & \\
& $\mathrm{kPa}(95 \% \mathrm{CI})$ & $332.2(306.5$ to 357.9$)$ & $473.0(434.7$ to 511.2$)$ & \\
\hline $\mathbf{3}$ & Level & Moderate & High & $4 \%$ \\
& $\mathrm{Z} \mathrm{score}(95 \% \mathrm{CI})$ & $0.52(0.37$ to 0.66$)$ & $2.49(2.32$ to 2.66$)$ & \\
& $\mathrm{kPa}(95 \% \mathrm{CI})$ & $334.9(242.9$ to 426.9$)$ & $800.6(607.7$ to 996.5$)$ & \\
\hline $\mathbf{4}$ & Level & High & High & \\
& $\mathrm{Z}$ score $(95 \% \mathrm{CI})$ & $2.69(2.53$ to 2.85$)$ & $1.54(1.37$ to 1.72$)$ & \\
& $\mathrm{kPa}(95 \% \mathrm{CI})$ & $425.2(331.1$ to 519.2$)$ & $592.8(452.9$ ot 732.7$)$ & \\
\hline
\end{tabular}

Table 5: Mean Z-scores (SD location relative to the mean of 0.00) of each independent variable across each of the 4 clusters. Parentheses are $95 \%$ confident limits and sample size of that comparison.

1: Bolded: significant difference $(\mathrm{p}<0.05)$ between the Moderate-Moderate PPDT class and the Low-Low PPDT class.

2: Mean location (z-score) is significantly below the mean $(0.00)$

3: Mean location (z-score) is significantly above the mean value $(0.00)$

\begin{tabular}{|c|c|c|c|c|}
\hline & Low-Low $(67 \%)$ & $\begin{array}{l}\text { Moderate-Moderate } \\
(25 \%)\end{array}$ & Moderate-High (4\%) & High-High (4\%) \\
\hline NDI & $0.16(0.08,0.24 ; 647)^{3}$ & $-0.33(-0.45,-0.21 ; 255)^{1,2}$ & $-0.07(-0.41,0.27 ; 30)$ & $-0.47(-0.71,-0.24 ; 41)^{1,2}$ \\
\hline TSK & $0.11(-0.02,0.24 ; 215)$ & $-0.16(-0.37,0.04 ; 93)$ & $-0.22(-0.80,0.40 ; 11)$ & $-0.35(-0.83,-0.13 ; 17)^{2}$ \\
\hline PCS & $0.12(0.01,0.24 ; 265)^{3}$ & $-0.33(-0.53,-0.14 ; 100)^{1,2}$ & $0.37(-0.28,1.01 ; 9)$ & $-0.14(-0.65,0.37 ; 15)$ \\
\hline NPRS & $0.11(0.04,0.18 ; 779)^{3}$ & $-0.25(-0.36,-0.14 ; 299)^{1,2}$ & $0.07(-0.21,0.35 ; 48)$ & $-0.19(-0.49,0.11 ; 44)$ \\
\hline SLANSS & $0.07(-0.05,0.18 ; 287)$ & $-0.10(-0.32,0.11 ; 86)$ & $-0.23(-0.71,0.24 ; 17)$ & $-0.36(-0.84,0.11 ; 18)$ \\
\hline IES & $0.07(-0.04,0.17 ; 338)$ & $-0.13(-0.31,0.04 ; 134)$ & $-0.16(-0.60,0.28 ; 20)$ & $-0.23(-0.52,0.38 ; 19)$ \\
\hline AROM & $-0.17(-0.27,-0.08 ; 392)^{2}$ & $0.37(0.22,0.52 ; 159)^{1,3}$ & $0.19(-0.20,0.59 ; 23)$ & $0.22(-0.20,0.63 ; 22)$ \\
\hline
\end{tabular}

Copyright (C) 2016 Wolters Kluwer Health, Inc. Unauthorized reproduction of the article is prohibited. 


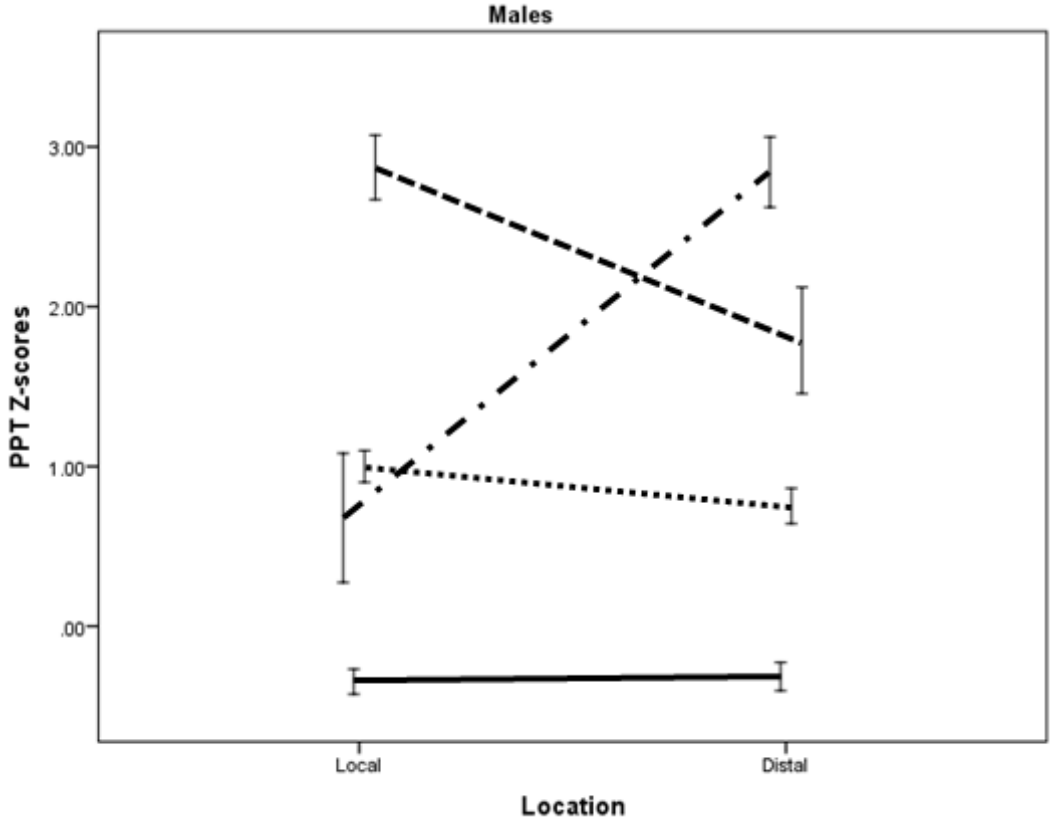

2

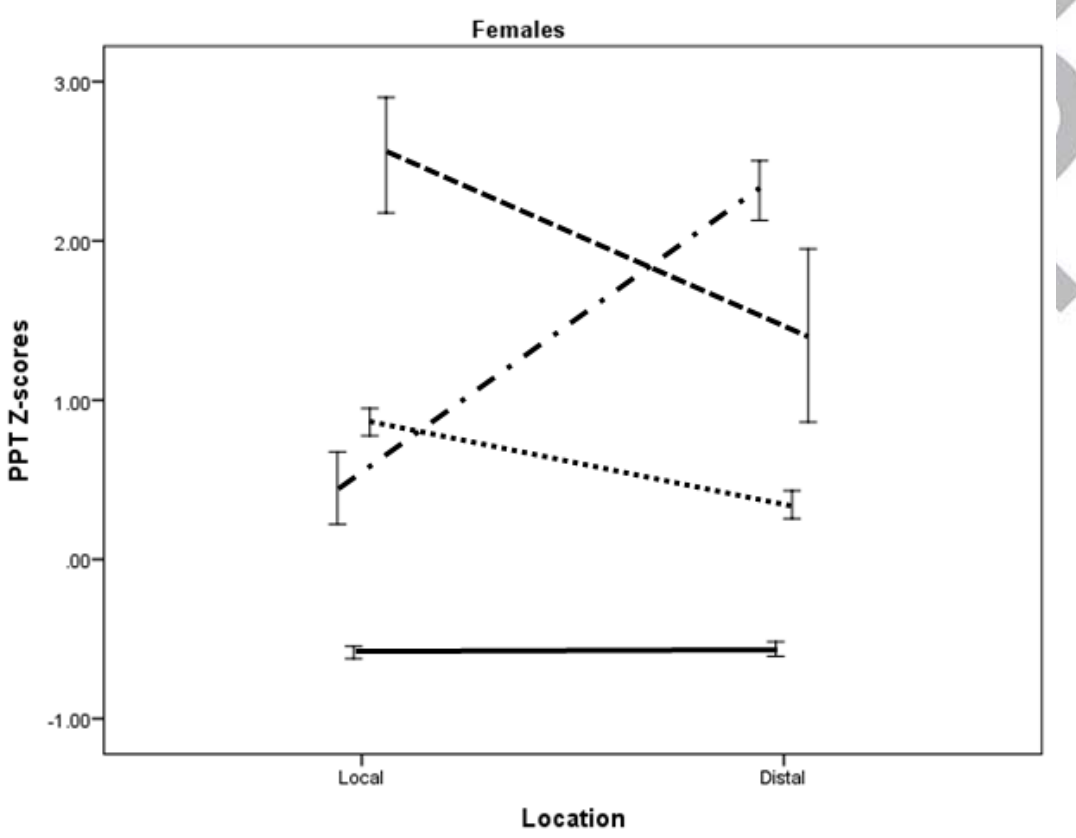

3
4
5
6

6 Figure $1 \mathrm{a}, \mathrm{b}$

Copyright (C) 2016 Wolters Kluwer Health, Inc. Unauthorized reproduction of the article is prohibited. 